\section{DGFF hat neuen Vorsitzenden}

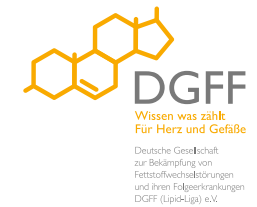

Bei der diesjährigen Mitgliederversammlung der Deutschen Gesellschaft zur Bekämpfung von Fettstoffwechselstörungen und ihren Folgeerkrankungen DGFF (Lipid-Liga) wurde Prof. Dr. Karl Winkler, Ärztlicher Direktor des Instituts für Klinische Chemie, Laboratoriumsmedizin - Lipidambulanz, Universitätsklinikum Freiburg, zum 1. Vorsitzenden der Fachgesellschaft gewählt.

$\mathrm{D}$ em neu gewählten Vorstand gehören außerdem an: Priv.-Doz. Dr. med. Volker Schettler (stellv. Vorsitzender), Göttingen, Prof. Dr. med. Hans-Ulrich Klör (stellv. Vorsitzender), Gießen, Prof. Dr. med. Peter Grützmacher (Schatzmeister), Frankfurt am Main, Dr. med. Martin Hulpke-Wette (Schriftführer), Göttingen, Prof. Dr. med. Stephan Jacob, Villingen-Schwenningen (Schriftleiter der Zeitschrift „Der Lipidreport“), Dr. med. Jens Ringel (Beisitzer), Potsdam,
Prof. Dr. med. Ulrich Julius (Beisitzer), Dresden und Dr. rer. biol. hum. Heike Kantner (Leiterin der Geschäftsstelle).

\author{
Weitere Informationen: \\ Deutsche Gesellschaft zur Bekämpfung von \\ Fettstoffwechselstörungen und ihren Folge- \\ erkrankungen DGFF (Lipid-Liga) e.V., \\ Bunsenstraße 5, 82152 Planegg bei München, \\ Tel.: 089/7191001, Fax: 089/7142687, \\ E-Mail: info@lipid-liga.de, \\ Internet: www.lipid-liga.de
}

\section{Schlafapnoe: Risikofaktor vor und nach Schlaganfall

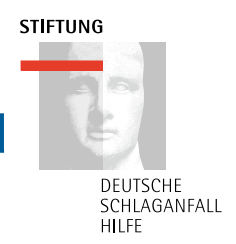

Verschiedene internationale Studien wiesen ein zwei- bis dreifach erhöhtes Schlaganfallrisiko bei Apnoe-Patienten aus.

$\mathrm{M}$ indestens 1-2\% der Bevölkerung ist vorsichtigen Schätzungen zufolge von einer Schlafapnoe betroffen. Die nächtlichen Atemstillstände führen mittelfristig zu Bluthochdruck, weil das Herz mehr pumpen muss, um die Organe ausreichend mit Sauerstoff zu versorgen. Hochdruck ist der Hauptrisikofaktor für einen Schlaganfall.

Studien aus Schweden und den USA lassen darauf schließen, dass auch die Schlafapnoe selbst das Risiko für einen Schlaganfall erhöht. Und dass nicht nur die besonders schweren Apnoen, sondern bereits leichte bis mittlere Syndrome den Schlaganfall fördern.

Wird die Schlafapnoe immer noch unterschätzt? „Vieles deutet darauf hin", meint Prof. Jean Haan, neurologischer Chefarzt der Kliniken Mariahilf in Mönchengladbach und Regionalbeauftragter der Stiftung Deutsche Schlaganfall-Hilfe. Haan hat festgestellt, dass Schlaganfälle offenbar zur Verschlechterung von Schlafapnoen führen. Doch in der Akutphase auf der Stroke Unit sind Patienten oft noch nicht stabil genug für eine Behandlung.

Sind Patienten erst einmal in die $\mathrm{Re}$ habilitation entlassen, „sieht man sie möglicherweise nie wieder", so Haan. „Hier liegt ein echtes Problem. Wenn es der Zustand des Patienten zulässt, sollte man ihn deshalb nicht ohne Anpassung an ein Druckbeatmungsgerät in die Reha entlassen." Er spricht sich dafür aus, bei Patienten mit schweren Schlaganfällen eine Schlafapnoe abzuklären. „Der Risikofaktor ist zu bedeutend, als dass man ihn vernachlässigen dürfte", so Haan. Eine schwere Apnoe kann außerdem den Rehabilitationserfolg gefährden, weil die Patienten müde und abgespannt sind.

Stiftung Deutsche Schlaganfall-Hilfe 